\title{
Thermal activation in statistical clusters of magnetic nanoparticles
}

\author{
O. Hovorka ${ }^{1}$ \\ Faculty of Engineering and the Environment, University of Southampton, Southampton, SO16 7QF, \\ $U K$
}

(Dated: 23 November 2016)

\begin{abstract}
This article presents a kinetic Monte-Carlo study of thermally activated magnetisation dynamics in clusters of statistically distributed magnetic nanoparticles. The structure of clusters is assumed to be of fractal nature, consistently with recent observations of magnetic particle aggregation in cellular environments. The computed magnetisation relaxation decay and frequency-dependent hysteresis loops are seen to significantly depend on the fractal dimension of aggregates, leading to accelerated magnetisation relaxation and reduction in the hysteresis loops size as the fractal dimension increases from one-dimensional-like to three-dimensionallike clusters. Discussed are implications for applications in nanomedicine, such as magnetic hyperthermia or magnetic particle imaging.
\end{abstract}

\section{Introduction}

Magnetic nanoparticles (MP) are widely considered for therapeutic and diagnostic (theranostic) technologies in nanomedicine. ${ }^{1}$ For example, magnetic particle hyperthermia for cancer therapy utilises MPs as local heat generators to bring cancerous tumours to cytotoxic temperatures. ${ }^{2}$ In magnetic particle imaging methodology for tumour imaging, MPs act as magnetic tracers and direct magnetic signal generators for detection, ${ }^{3,4}$ while they have indirect function as contrast agents in magnetic resonance imaging. ${ }^{5}$ Magneto-relaxometry is a promising technique for bio-sensing and detection, with a potential for simultaneous identification of several biomarkers for efficient and unique diagnosis of disease. ${ }^{6,7}$

Development of theranostic technologies relies on the availability of MPs optimised to achieve high specific heat rates and resolution in sensing and imaging. This drives the search for new material processing protocols for MPs with high degree of control of shape, statistical distributions of size, magneto-crystalline anisotropy, etc. ${ }^{2,4,8}$ The research emphasis also shifts towards the MPs with a core-shell structure, ${ }^{9}$ and multifunctional and hybrid nanostructures. ${ }^{10,11}$ In addition, the practical development of applications requires addressing important issues related to MP standardisation ${ }^{12}$, and identifying chemical synthesis routes towards up-scaling the MP production to industrial scales. ${ }^{8}$

However, growing evidence suggests that the performance of even well optimised MPs becomes considerably degraded when MPs are immersed in biological environments, such as during internalisation in living cells. ${ }^{13,14}$ MP aggregation is believed to be the primary cause behind these observations where, as a consequence of the magnetostatic and possibly exchange interactions, the details of arrangement and statistical distributions of MPs within aggregates play a dominant role in determining the overall thermal magnetisation dynamics. ${ }^{15-18}$ Despite the fact that our understanding of the fundamental issues related to the symbiosis between magnetic nanostructures and biological environments is still rather limited, recent transmission electron microscopy imaging experiments suggest that the kinetics of intracellular aggregation processes leads to MP aggregates of statistically fractal nature. ${ }^{15,16}$ This finding provides a pathway for systematic explorations of the relationship between realistic MP aggregate cluster structures as may be observed in living cells, and their thermal fluctuation-driven magnetisation dynamics relevant in theranostic applications.

This work presents computational investigation of the effect of statistically distributed fractal MP clusters on their thermally activated magnetisation behaviour. Given that the MP aggregates become immobile during the internalisation in living cells, and the relatively long microsecond-to-second timescales relevant in theranostic applications, the Néel-Arrhenius thermal relaxation is the dominant mechanism driving magnetic thermal fluctuations. This allows for the application of the kinetic-Monte-Carlo (kMC) approach with realistic timescales. ${ }^{19-21}$ The kMC model includes dipolar interactions between the MPs within clusters, and naturally incorporates thermal fluctuations of Néel-Arrhenius type. We show that as the MP cluster geometry varies from low fractal dimension, which corresponds to disordered chainlike structures, to high fractal dimension, corresponding to spherical clusters, the magnetisation behaviour evolves towards accelerated relaxation and reduced hysteresis loop size. This is a consequence of dipolar interactions within MP clusters, and implies degraded theranostic performance in comparison to non-interacting MP assemblies.

The article is organised as follows. Section 2 introduces the kMC method and a summary of the used simulation parameters. Section 3 introduces basic concepts and the algorithm used for generating the ensembles of fractal clusters, including the structural quantification of their geometrical properties. Section 4 is dedicated to the discussion of magnetic relaxation in ensembles of fractal clusters. Section 5 addresses the issue of the hysteresis loop behaviour in ensembles of cluster structures for different applied field amplitudes and frequency. Sections 6 and 7 present the discussion of the results with relevance to theranostics. 


\section{Modelling methodology}

\subsection{Interacting Stoner-Wohlfarth particles}

Fig. 1(a) illustrates typical MP cluster structures considered in this work. MPs inside clusters are assumed to be Stoner-Wohlfarth-like particles. A typical cluster contains $N$ spherical particles of radius $a$ and volume $V=4 \pi a^{3} / 3$, composed of a magnetic core of radius $a_{m} \leq a$ and a non-magnetic shell of thickness $a-a_{m}$. Magnetic moments of MPs are $\vec{m}_{i}=M_{s} V_{m} \hat{m}_{i}$, where $M_{s}$ is the saturation magnetisation, $V_{m}=4 \pi a_{m}^{3} / 3$ is the magnetic volume of a MP, and $\hat{m}_{i}$ is the magnetic moment vector of unit length. The uniaxial anisotropy vector is denoted as $\vec{K}_{i}=K \hat{k}_{i}$. Throughout this study MPs are assumed to have identical volume characteristics $V_{m}, V$ and the anisotropy constant $K$, and spherically random distribution of anisotropy axes $\hat{k}_{i}$. Thus besides the randomised fractal-like arrangement of MPs inside the clusters, to be discussed below (Fig. 1(a)), the spherical anisotropy axis distribution is the only source of randomness.

The governing energy density associated with a MP cluster reads:

$$
\frac{E}{2 K V_{m}}=\sum_{i=1}^{N}\left(\frac{1}{2}\left(\hat{k}_{i} \times \hat{m}_{i}\right)^{2}-\hat{m}_{i} \cdot \vec{h}-\hat{m}_{i} \cdot \vec{h}_{i}^{\text {dip }}\right)
$$

The first anisotropy energy term determines the preferential orientation of MP moments $\hat{m}_{i}$ towards their anisotropy vectors $\hat{k}_{i}$. The second term is the Zeeman energy, where $\vec{h}=h \hat{h}$ is the external field vector of strength $h=H / H_{K}$ normalised by anisotropy field $H_{k}=2 K / M_{s}$. The last term is the dipolar interaction energy due to all neighbouring particles of $\hat{m}_{i}$ inside the cluster with:

$$
\vec{h}_{i}^{\mathrm{dip}}=I \sum_{j \neq i} r_{i j}^{-3}\left(-\hat{m}_{j}+3 \hat{r}_{i j}\left(\hat{m}_{j} \cdot \hat{r}_{i j}\right)\right)
$$

where $\hat{r}_{i j}=\vec{r}_{i j} / r_{i j}, \quad r_{i j}$ is the particle-particle distance normalised by the particle radius $a$, and $I=$ $2 \pi M_{s} V_{m} /\left(3 H_{K} V\right)$ is the dipolar interaction strength.

Stable magnetic states $\alpha$ of a MP cluster, i.e. specific realisations of magnetic moments $\hat{m}_{i}$ of all particles inside a cluster, correspond to energy minima $E_{\alpha}$ found by minimisation of Eq. (1). The transition rates $w_{\alpha \beta}$ for thermally activated transitions from a state $\beta$ to a state $\alpha$ are specified by the Néel-Arrhenius law:

$$
w_{\alpha \beta}=f_{0} \exp \left(-\Delta E_{\alpha \beta} / k_{B} T\right)
$$

where $\Delta E_{\alpha \beta}=E_{\text {saddle }}-E_{\beta}$ is the energy barrier, and $E_{\text {saddle }}$ is the saddle point energy along the transition path from $\beta$ to $\alpha$. The maximum number of states in a cluster composed of $N$ particles with uniaxial anisotropy is $2^{N}$, which corresponds to the maximum $N(N-1)$ of energy barriers. These physical notions are fundamental to the description of thermally activated magnetisation dynamics outlined next.
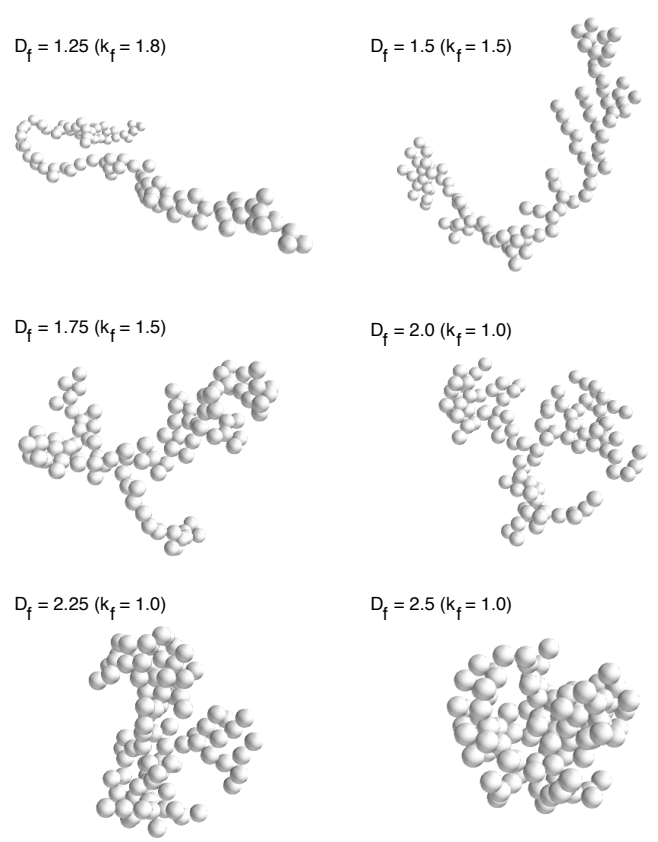

$D_{f}=2.75\left(k_{f}=1.0\right)$

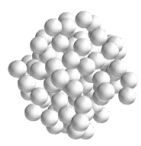

$D_{f}=3.0\left(k_{f}=1.0\right)$

(a)
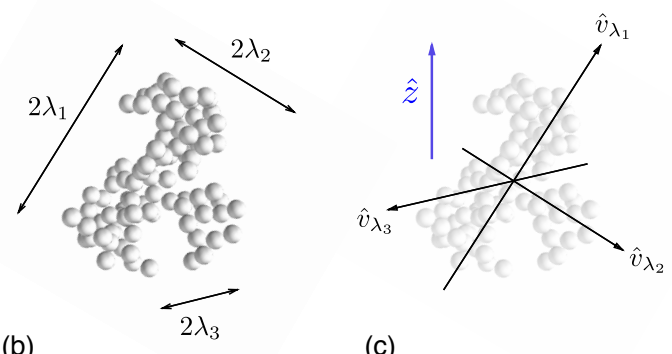

(c)

FIG. 1. (a) Examples of cluster structures of $N=100$ particles of different fractal dimension $D_{f}$, generated using the algorithm described in Section 3 and obeying the scaling relation in Eq. (5). (b) Eigenvalues of the gyration tensor Eq. (8) are ordered such that $\lambda_{1} \geq \lambda_{2} \geq \lambda_{3}$ and are related to the characteristic size of the cluster along the principal directions defined by the eigenvectors $\hat{v}_{\lambda_{1}}, \hat{v}_{\lambda_{2}}, \hat{v}_{\lambda_{3}}$ illustrated in (c). The action of the applied field $\vec{h}$ on the cluster can be quantified with respect to the principal direction vectors $\hat{v}_{\lambda_{1}}$, $\hat{v}_{\lambda_{2}}, \hat{v}_{\lambda_{3}}$.

\subsection{Kinetic Monte-Carlo model}

A direct integration of the Landau-Lifshitz-Gilbert dynamics to study the thermal fluctuation-driven magnetisation behaviour in MPs becomes computationally inefficient as the size and anisotropy of MPs increase. This is 
due to the increasing energy barriers $\Delta E_{\alpha \beta}$, which lead to reduced thermal activation rates $w_{\alpha \beta}$ to a computationally inaccessible dynamical range. An alternative approach, shown to be valid in the limit of high energy barriers and large damping, has been based on the socalled discrete orientation model. ${ }^{22,23}$ In the discrete orientation model, the Fokker-Planck equation representing the stochastic Landau-Lifshitz-Gilbert dynamics is consistently replaced by a Master Equation describing thermal fluctuations as Néel-Arrhenius hopping process through discrete states $\alpha$ and with transition rates $w_{\alpha \beta}$ :

$$
\frac{d P_{\alpha}}{d t}=\sum_{\beta}\left(w_{\alpha \beta} P_{\beta}-w_{\beta \alpha} P_{\alpha}\right)
$$

where $P_{\alpha}$ are the probabilities of states $\alpha$ at a given time, and the sum extends through all states that can be formed by the stable MP moment configurations in a cluster as described in Section 2.1. Eq. (4) is a set of coupled first order differential equations subject to initial condition $P_{\alpha}(0){ }^{24}$

Generally speaking, kinetic Monte-Carlo (kMC) is a method to solve Eq. (4) in a stochastic manner. ${ }^{25}$ The present work builds on the reduced kMC methodology developed recently to study long-time thermally activated behaviour in large assemblies of MPs. ${ }^{19-21,26}$ The key assumption in this approach is the statistical likelihood of only single-particle transitions, which is reasonable in the weakly interacting limit. Then, a transition from a state $\beta$ to $\alpha$ can be uniquely associated with an abrupt reversal of only a single MP moment. ${ }^{27}$ This allows the approximation of the energy barrier $\Delta E_{\alpha \beta}$ by a single particle term in energy of Eq. (1) assuming the MP moment configurations consistent with the starting state $\beta$. This reduced procedure avoids the problem of finding energy barriers by complex optimisation methods required for multidimensional energy landscapes, and allows efficient computations over thousands of interacting MPs and beyond. The entire approach has been validated for small systems of interacting MPs using semianalytical calculations. ${ }^{28}$

\subsection{Summary of simulation parameters}

The analysis of thermally activated magnetisation behaviour presented below assumes ensembles of 100 clusters each containing $N=100 \mathrm{MPs}$ in contact, i.e. 10000 particles in total per ensemble. The clusters in every ensemble have the same fractal dimension but the random realisation of MPs varies from cluster to cluster. Several different ensembles will be considered in the analysis assuming fractal dimensions $D_{f}=1.25,1.5,1.75,2$, $2.25,2.5,2.75$, 3, with examples of characteristic structures shown in Fig. 1. The outer particle radius is set to $a=15 \mathrm{~nm}$, inner magnetic radius to $a_{m}=10 \mathrm{~nm}$, $M_{s}=400 \mathrm{emu} / \mathrm{cm}^{3}$ and $K=2 \times 10^{5} \mathrm{erg} / \mathrm{cm}^{3}$ resembling $\mathrm{Fe}_{3} \mathrm{O}_{4}$ particles, which gives $H_{K}=1 \mathrm{kOe}$ and $I \approx 0.62 M_{s} / H_{K} \approx 0.245$. Two perpendicular orientations of the external field will be considered, along the $\hat{z}$ and $\hat{x}$ axis of the coordinate system, i.e. $\vec{h}=h \hat{z}$ and $\vec{h}=h \hat{x}$, respectively. The hysteresis loop calculations will use sinusoidal external field with amplitudes $H_{m}=h_{m} H_{K}=2 \mathrm{kOe}$ (major loop) and 300 Oe (minor loop), and applied field frequencies $f_{h}=1 \mathrm{~Hz}$ and $100 \mathrm{kHz}$. The field amplitude 300 Oe is chosen to represent the maximum field amplitude of the most magnetic hyperhermia experimental systems operating at 100 $\mathrm{kHz}^{2,29,30}$ The evaluation of thermal transition rates using Eq. (3) will assume constant attempt frequency $f_{0}=10^{9} \mathrm{~Hz}$, as standard, and temperature $T=300$ $\mathrm{K}$.

\section{Clusters of MPs with fractal geometry}

\subsection{Basic definitions}

The fractal clusters of $N$ particles of radius $a$, such as illustrated in Fig. 1(a), satisfy the following scaling relation:

$$
N=k_{f}\left(\frac{R_{g}}{a}\right)^{D_{f}}
$$

where $k_{f}$ is a constant prefactor, $D_{f}$ is a fractal dimension, and $R_{g}$ is the radius of gyration of a cluster defined as:

$$
R_{g}^{2}=\frac{1}{N} \sum_{i=1}^{N}\left(\vec{r}_{i}-\vec{r}_{N}^{0}\right)^{2}
$$

where the $\vec{r}_{N}^{0}=N^{-1} \sum_{i=1}^{N} \vec{r}_{i}$ is the centre of mass of $N$ particles. Alternatively, equivalent definition of $R_{g}$ can be given as:

$$
R_{g}^{2}=\lambda_{1}^{2}+\lambda_{2}^{2}+\lambda_{3}^{2}
$$

where $\lambda_{1}, \lambda_{2}, \lambda_{3}$ are the eigenvalues of the matrix representing the gyration tensor:

$$
S_{m n}=\frac{1}{N} \sum_{i=1}^{N}\left(\vec{r}_{i}-\vec{r}_{N}^{0}\right)_{m}\left(\vec{r}_{i}-\vec{r}_{N}^{0}\right)_{n}
$$

where the indices $m, n$ refer to $x, y, z$ coordinate components. The eigenvalues $\lambda_{k}, k=1,2,3$, represent the characteristic average mean-square size of a cluster along the three principal directions defined by the eigenvectors $\hat{v}_{\lambda_{k}}$ associated with $\lambda_{k}$, as illustrated in Figs. 1(b)-(c).

\subsection{Fractal cluster generating algorithm}

The algorithm to generate MP cluster structures with fractal geometries consistent with the experimental observations of MP aggregates in cellular environments ${ }^{15}$, and satisfying the fractal scaling Eq. (5), has been developed earlier ${ }^{31}$ and can be outlined as follows. To initialise the algorithm to produce a particle cluster with specific values of $k_{f}$ and $D_{f}$, place the first two particles in contact along the $\hat{z}$ axis of the coordinate system. Generate randomly the coordinates of the next particle such that it is in contact with an already existing particle and it 

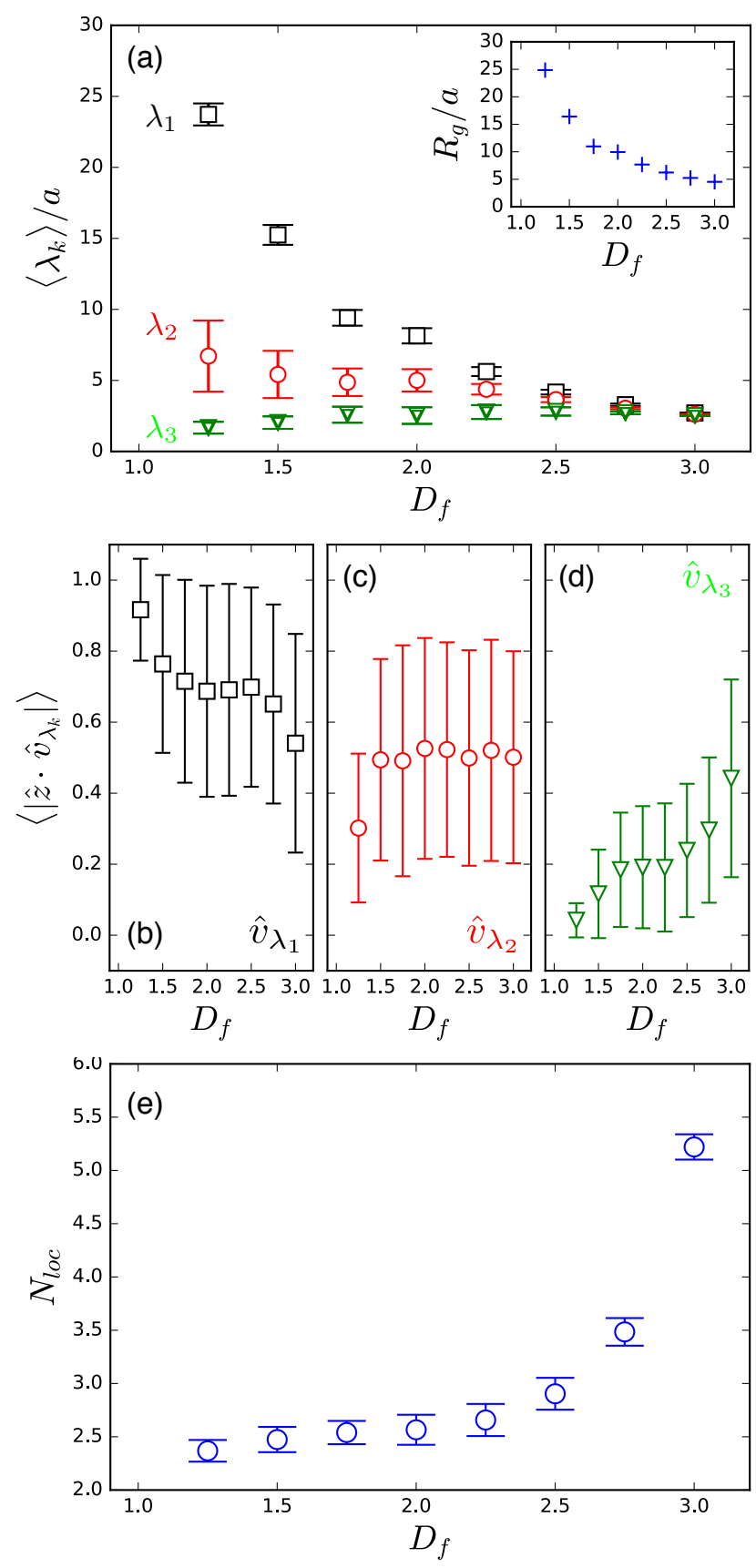

FIG. 2. (a) Ensemble averaged eigenvalues $\lambda_{1} \geq \lambda_{2} \geq \lambda_{3}$ of the gyration tensor matrix $S$ as a function of the cluster fractal dimension $D_{f}$. The normalisation is by the particle radius $a$. Inset: Dependence of the normalised radius of gyration $R_{g} / a$ on $D_{f}$. (b)-(d) Ensemble averaged projection of eigenvectors $\hat{v}_{\lambda_{k}}$ onto the $\hat{z}$ axis of the coordinate system as illustrated in Fig. 1(c). (e) Local number of neighbouring particles of a particle as a function of $D_{f}$.

obeys Eqs. (9)-(10) below with $L=3$ : more generally, assuming that $L-1$ particles in a cluster, with particles labeled as $1,2, \ldots, L, \ldots N$, have already been iteratively generated, add a new particle $L$ such that:
1) The centre of the new particle $L$ is situated on the surface of a 'large sphere' of radius $R$ :

$$
\left(\vec{r}_{L}-\vec{r}_{L-1}^{0}\right)^{2}=R^{2}
$$

where $\vec{r}_{L-1}^{0}$ is the centre of mass of the existing $L-1$ particles calculated as in Eq. (6), and the radius $R$ is given by:

$$
\begin{aligned}
R^{2}= & \frac{L^{2} a^{2}}{L-1}\left(\frac{L}{k_{f}}\right)^{2 / D_{f}} \\
& -\frac{L a^{2}}{L-1}-L a^{2}\left(\frac{L-1}{k_{f}}\right)^{2 / D_{f}}
\end{aligned}
$$

Eq. (10) guarantees consistency with the fractal scaling law defined by Eq. (5). ${ }^{31}$

2) The new particle $L$ is in contact with at least one of the existing $L-1$ particles and there is no overlap between the new particle and the existing particles. The conditions to achieve this can be obtained by using straightforward geometrical arguments. The procedure outlined in 1) and 2) is iteratively repeated until all of the $N$ particles have been generated and the full fractal cluster structure grown.

\subsection{Structural characterisation of fractal clusters}

The cluster-generating algorithm introduced above is used next to generate ensembles of clusters of variable fractal dimension $D_{f}$. Every ensemble for a given $D_{f}$ in the present study assumes 100 clusters with the same $D_{f}$ and containing $N=100 \mathrm{MPs}$ (Fig.1(a)), i.e. total 10000 MPs per ensemble. The algorithm guarantees the fractal scaling of Eq. (5) for all clusters. The positions of MPs inside clusters and the orientation of the individual clusters in an ensemble will differ from cluster to cluster in a randomised manner. The magnetisation behaviour of an ensemble is expected to depend on the overall effective shape of clusters and the relative orientation of clusters with respect to the applied field. Thus the statistical distributions of the cluster shape and orientation, as they are generated by the cluster-generating algorithm, need to be quantified to allow consistent analysis of their magnetisation behaviour. These characteristics can be assessed by studying the eigenvalues $\lambda_{k}$ of the gyration tensor defined in Eq. (8), and the projections of the corresponding eigenvectors $\hat{v}_{\lambda_{k}}$ onto the $\hat{z}$ direction of the coordinate system (Figs. 1(b)-(c)).

Fig. 2(a) shows the $D_{f}$ dependence of the average eigenvalues $\left\langle\lambda_{1}\right\rangle \geq\left\langle\lambda_{2}\right\rangle \geq\left\langle\lambda_{3}\right\rangle$ normalised by the particle radius $a$. The averaging was performed by first sorting the eigenvalues for each cluster in an ensemble and finding the mean of the largest, intermediate and smallest eigenvalues, respectively. The dependence of the radius of gyration on $D_{f}$ evaluated by Eq. (7) for every ensemble is also shown in the inset in Fig. 2(a), and confirms the hyperbolic dependence suggested by Eq. (5). Similarly, Figs. 2(b)-(d) show the relative orientation of clusters obtained by evaluating the projections of the 
eigenvectors $\hat{v}_{\lambda_{k}}$ onto the $\hat{z}$ axis of the coordinate system (Fig. 1(c)). The eigenvector projections were ordered according the magnitudes of their corresponding eigenvalues and averaged over all clusters in an ensemble. The extent of the errorbars of the projections quantifies the amount of the orientational variation: small errorbars suggest that all clusters are well aligned, while large errorbars imply significant variation in the alignment.

As can be seen in Fig. 2(a), one eigenvalue is dominant for clusters with low $D_{f} \rightarrow 1$, which suggests the presence of locally one-dimensional-like randomised structures in the ensemble, as expected according to Fig. 1(a). The projection of $\hat{v}_{\lambda_{1}}$ is also the largest, and the errorbars of $\hat{v}_{\lambda_{1}}, \hat{v}_{\lambda_{2}}, \hat{v}_{\lambda_{3}}$ are non-overlapping, implying that these locally chain-like structures in the ensemble are statistically preferentially aligned along the $\hat{z}$ axis of the coordinate system. In the opposite extreme $D_{f} \rightarrow 3$, all eigenvalues become similar, confirming the spherical-like structure of clusters in the ensemble (Fig. 1(a)). The eigenvector projections and the extent of errorbars in Figs. 1(b)-(d) become practically indistinguishable due to the spherical symmetry. In the intermediate region around $D_{f} \sim 2$, the projections of $\hat{v}_{\lambda_{1}}$ and $\hat{v}_{\lambda_{2}}$ corresponding to the two larger eigenvalues dominate the projection of $\hat{v}_{\lambda_{3}}$, suggesting a locally planar structure of clusters statistically oriented along the $\hat{z}$ coordinate axis.

The preferential structural orientation of MP clusters along the $\hat{z}$ direction observable in ensembles with lower $D_{f}$ appears to be the result of the specific implementation of the cluster-generating algorithm in Section 3.2, where the first two MPs during the cluster growth are placed along the $\hat{z}$ axis. While this initial condition seems to set the preferential direction for the cluster growth, it has no effect on the fractal structure of clusters because placing the first two particles in contact along some global direction does not influence the relative positions of the remaining MPs in the local coordinate system of a cluster.

The choice of initialisation of the cluster growth along the $\hat{z}$ direction thus seems to mimic the effect of the applied field aiding the preferential alignment of MPs during the aggregation process. However, since the update rules in the cluster-generating algorithm in Section 3.2 are independent of the applied field, this hypothesis will need to be validated in future studies by assessing the effects of the applied field acting during the MP aggregation on empirical values of $k_{f}$ and $D_{f}$, i.e. the values of $k_{f}$ and $D_{f}$ emerging in the long-time limit of the MP aggregation process subject to applied field. It is interesting to note, that the experimental observations of fractal structures of MP aggregates inside living cells gave $k_{f} \sim 1.33-1.85$ and $D_{f} \sim 1.96-2.03,{ }^{15}$ thus motivating the investigations of ensembles of clusters with the same $D_{f}$ such as in this work. The present study considers a significantly broader $D_{f}$ range of fractal structures, as shown Fig. 1(a), to allow for broader comparative analysis and the assessment of the effects of fractal structures on thermally activated magnetisation behaviour in MP
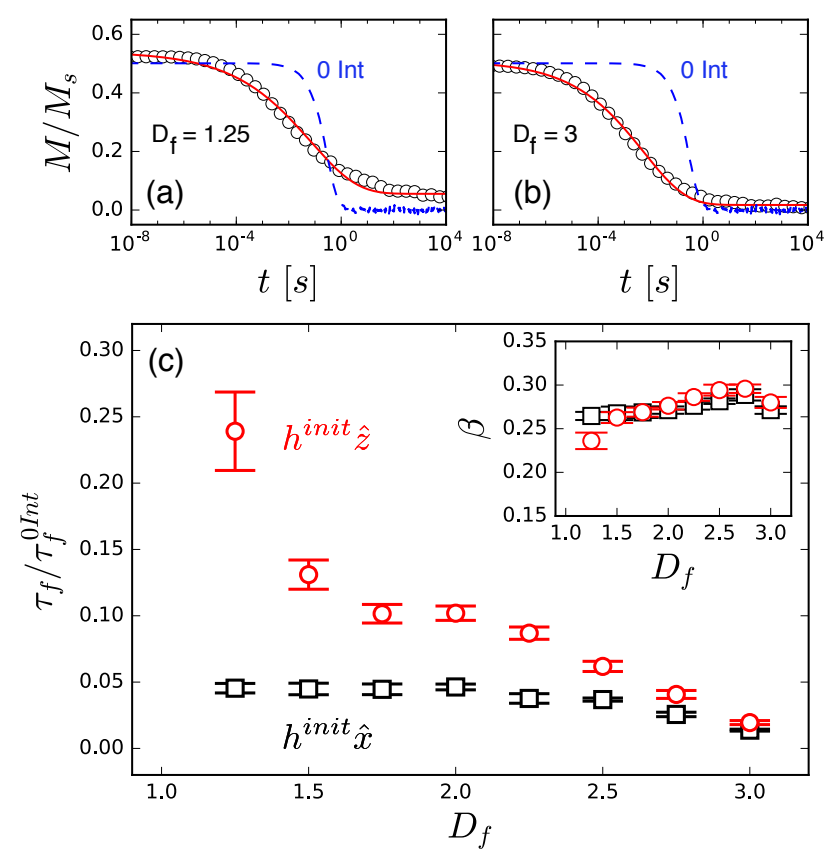

FIG. 3. Magnetisation relaxation decay profile for ensembles of clusters of fractal dimension (a) $D_{f}=1.25$ and (b) $D_{f}=3$, suggesting that relaxation accelerates as $D_{f}$ increases. Circles correspond to kinetic Monte-Carlo simulations as described in Section 2.3. The solid line through the points is the fit of the stretched exponential Eq. (11). The dashed line corresponds to the non-interacting case and is independent of $D_{f}$. (c) Fractal dimension dependence of the characteristic relaxation time scale $\tau_{f}$ obtained from fitting the stretched exponential to various magnetisation decay profiles in ensembles of clusters shown in Fig. 1(a). Two initialisations of relaxation are considered, in perpendicular fields $\vec{h}=h^{i n i t} \hat{z}$ and $h^{i n i t} \hat{x}$.

systems. The preferential alignment of clusters inside cells under different experimental conditions has not yet been systematically studied.

It is also important to emphasise that due to the fractal nature of clusters their structure needs to be interpreted locally, i.e. with respect to the neighbours of a particle, as the MP clusters for any $D_{f}$ are expected to be effectively three-dimensional on longer length-scales (Fig. 1(a)). This is supported in Fig. 2(e), which shows the dependence of the average number of neighbours of a particle, $N_{l o c}$, on $D_{f}$, obtained by first counting all neighbours of a particle within the centre-to-centre distance $1.2 \times(2 a)$, where $2 a$ is the particle diameter. As shown, the number of neighbours per particle increases from $N_{l o c} \sim 2$ for $D_{f} \rightarrow 1$ (locally chain-like structure) to $N_{l o c} \sim 6$ for $D_{f} \rightarrow 3$ (locally spherical-like structure), as expected.

\section{Magnetic relaxation}

The analysis in the previous section quantified the characteristic statistical properties of ensembles of clusters of variable $D_{f}$ obtained by the cluster-generating algorithm introduced in Section 3.2. For low $D_{f} \rightarrow 1$, the clus- 
ters within ensembles have been found to be randomised chain-like structures with the largest characteristic size aligned along the $\hat{z}$ axis of the coordinate system. In the intermediate range $D_{f} \sim 2$, clusters are more planar-like oriented along the $\hat{z}$ axis, progressing towards spherical clusters for $D_{f} \rightarrow 3$. This now allows to investigate the magnetisation behaviour in the ensembles of these clusters and its dependence on $D_{f}$.

First consider the magnetic relaxation in zero applied field. The initial state for relaxation is prepared by rapidly reducing the external field $\vec{h}^{\text {init }}$ from saturation to zero by setting a high field rate in the kinetic MonteCarlo algorithm introduced in Section 2. Two different cases of the initialisation field will be considered, namely the field orientation along the $\hat{z}$ and $\hat{x}$ axes of the coordinate system, i.e. $\vec{h}^{\text {init }}=h^{\text {init }} \hat{z}$ and $\vec{h}^{\text {init }}=h^{\text {init }} \hat{x}$ respectively. After the initialisation, the external field is set to zero, $h=0$, during the subsequent magnetisation decay process.

Figs. 3(a) and (b) show the magnetisation relaxation decay for ensembles of clusters with $D_{f}=1.25$ and $D_{f}=3$ obtained after the initialisation of relaxation in $\vec{h}^{\text {init }}=h^{\text {init }} \hat{z}$. For comparison, shown is also the magnetisation relaxation decay of the equivalent noninteracting MP system, which is independent of $D_{f}$. Initial magnetisation is close to 0.5 , as expected for particle assemblies with spherically random anisotropy axes. To identify the characteristic relaxation time scale, we fit to the data the stretched exponential: ${ }^{32}$

$$
M(t)=M_{0} \exp \left(-\left(t / \tau_{f}\right)^{\beta}\right)
$$

where the fit constant $\tau_{f}$ gives an estimate of the characteristic time and $\beta$ is the stretching exponent. The fit to the non-interacting data gave $\tau_{f} \equiv \tau_{f}^{0 I n t}=298 \pm 10$ ms and $\beta=1.03 \pm 0.005$, which confirms the expected single exponential nature of the magnetisation decay in ensembles of identical MPs. The obtained value of $\tau_{f}^{0 I n t}$ agrees closely with the estimate $0.308 \mathrm{~ms}$ obtained from the Arrhenius law $\tau=\tau_{0} \exp \left(K V_{m} / k_{B} T\right) / 2$, assuming MPs with $K=2 \times 10^{5} \mathrm{erg} / \mathrm{cm}^{3}, a_{m}=10 \mathrm{~nm}, T=300 \mathrm{~K}$, and $\tau_{0}=10^{-9} \mathrm{~s}$, which corroborates the kinetic MonteCarlo simulations introduced in Section 2.3.

The fit of the stretched exponential to the interacting magnetisation decay with $D_{f}=1.25$ shown in Fig. 3(a) gave the values $\tau_{f}=71.2 \pm 8.8 \mathrm{~ms}$ and $\beta=$ $0.236 \pm 0.0094$. Thus $\tau_{f}$ is reduced in comparison with the non-interacting case, i.e. thermal relaxation accelerates due to the presence of particle-particle interactions within clusters in the ensemble. The reduced value of the exponent $\beta$ and the longer tail in comparison to the non-interacting case suggests that the functional profile of the magnetisation decay takes a more complex form beyond the single exponential decay observed in the noninteracting case.

Fig. 3(b) shows similar behaviour of the magnetisation decay in an ensemble of MP clusters with $D_{f}=3$. The stretched exponential fits yielded $\tau_{f}=5.8 \pm 0.45$

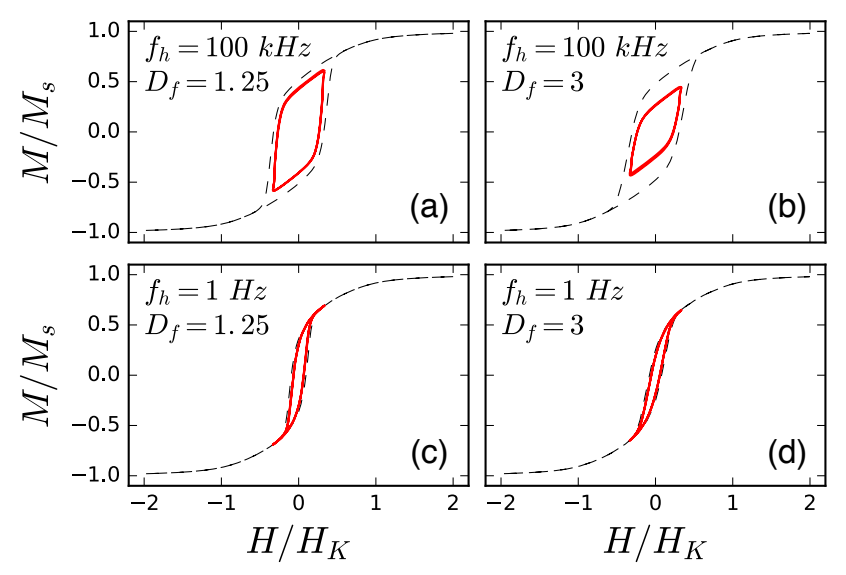

FIG. 4. Hysteresis loops obtained from the kinetic MonteCarlo modelling of cluster ensembles such as shown in Fig. 1 for fractal dimension (a), (c) $D_{f}=1.25$ and (b), (d) to $D_{f}=$ 3 , and sinusoidal applied field frequencies $f_{h}=100 \mathrm{kHz}$ and $1 \mathrm{~Hz}$. The major hysteresis loops (dashed line) correspond to external field amplitude $2 \mathrm{kOe}$ and the minor hysteresis loop (solid line) to amplitude 300 Oe.

ms and $\beta=0.280 \pm 0.0063$, i.e. relaxation accelerating even further and only a weak sensitivity of the stretching exponent. These differences are the manifestation of the interaction effects.

Fig. 3(c) shows the $D_{f}$ dependence of the characteristic relaxation time $\tau_{f}$ identified from the fits of the stretched exponential to the magnetisation decay profiles initialised in $\vec{h}^{\text {init }}=h^{\text {init }} \hat{z}$ for all ensembles of clusters generated earlier (Figs. 1, 2). It confirms the trend of the accelerating relaxation with increasing $D_{f}$. Similar behaviour is seen after the initialisation of the relaxation process in perpendicular field $\vec{h}^{\text {init }}=h^{\text {init }} \hat{x}$, although the dependence is weaker. For cluster structures with high $D_{f}$, the differences between the relaxation profiles for the two different initialisations diminish due to the increased symmetry when the directional dependence of the initialisation field $\vec{h}^{\text {init }}$ becomes irrelevant, which is in agreement with previous studies. ${ }^{28}$ The behaviour of the stretching exponent $\beta$ is shown in the inset in Fig. $3(\mathrm{c})$ and appears to be only weakly dependent on $D_{f}$ and on initialisation, which suggest relative robustness of the functional form of the relaxation decay profiles in the different cases of ensembles.

\section{Time-dependent external field}

\subsection{Hysteresis loops}

Hysteresis loops were studied using the kinetic MonteCarlo modelling using a sinusoidal external field $h=$ $h_{m} \sin \left(2 \pi f_{h}\right)$ oriented along the $\hat{z}$ axis, $\vec{h}=h \hat{z}$, and assuming two fixed frequencies $f_{h}=100 \mathrm{kHz}$ and $f_{h}=1$ $\mathrm{Hz}$.

Fig. 4 shows examples of a major (dashed line) and minor (solid line) hysteresis loop corresponding to field amplitudes $h_{m} H_{k}=2 \mathrm{kOe}$ and 300 Oe, respectively, for 

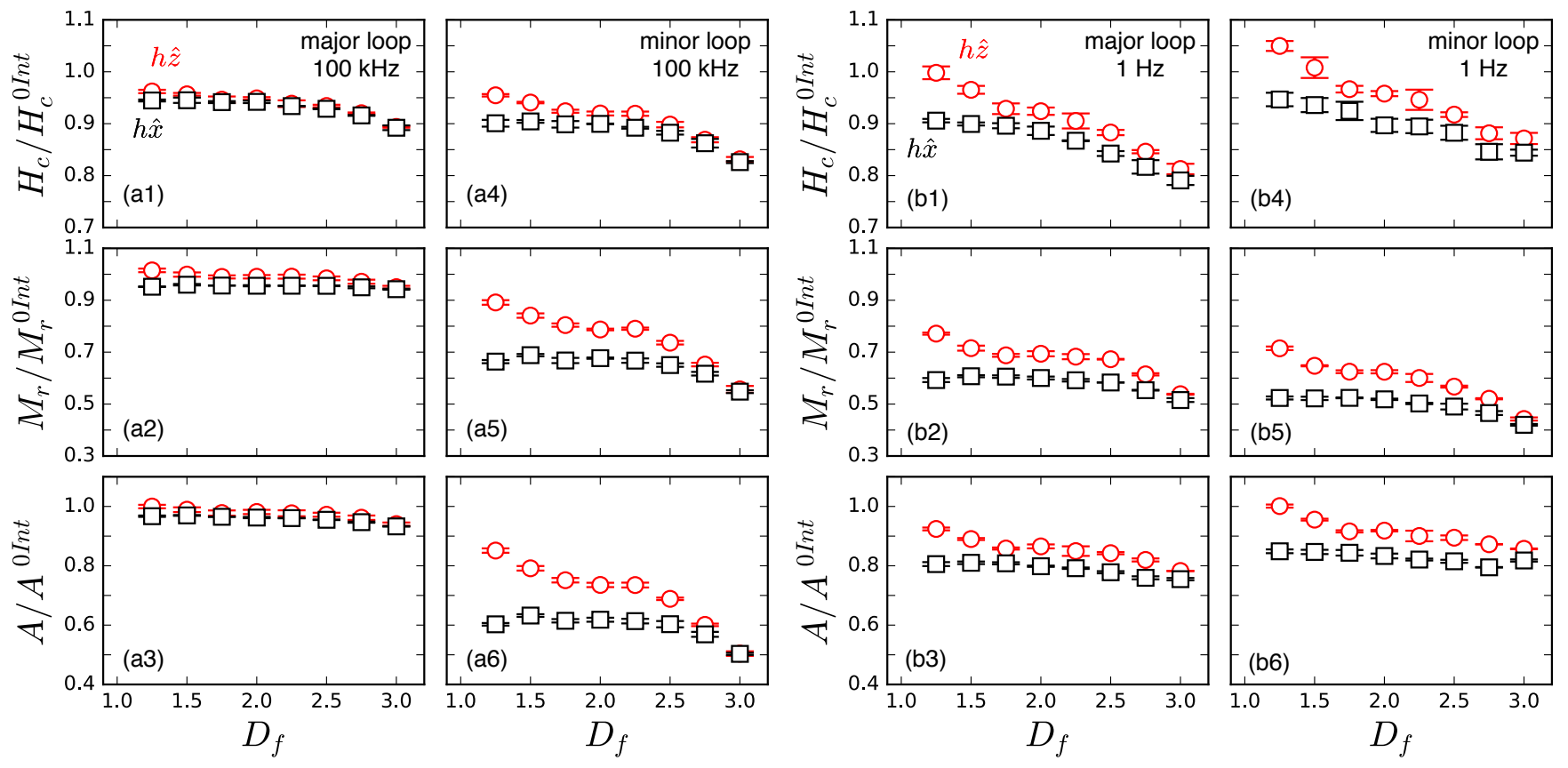

FIG. 5. Quantification of the cluster fractal dimension $D_{f}$ dependence of hysteresis loop characteristics: coercive field $H_{c}=$ $H_{K} h_{c}$, remanent magnetisation $M_{r}$, and hysteresis loop area $A$, normalised by their non-interacting system counterparts $H_{c}^{0 I n t}$, $M_{r}^{0 I n t}, A^{0 I n t}$. Subfigures (a1)-(a6) correspond to applied field frequency $100 \mathrm{kHz}$, and (b1)-(b6) to $1 \mathrm{~Hz}$. Subfigures (a1)-(a3) and (b1)-(b3) correspond to major hysteresis loop with saturating field $2 \mathrm{kOe}$, (a4)-(a6) and (b4)-(b6) correspond to minor hysteresis loops with field amplitude 300 Oe, such as shown in Fig. 4. The circle and square symbols correspond respectively to perpendicular external field orientations $\vec{h}=h \hat{z}$ and $\vec{h}=h \hat{x}$.

$D_{f}=1.25$ and 3 . The external field frequency of 100 $\mathrm{kHz}$ is comparable with the fast time scales of the onset thermal relaxation (Fig. 3(a)-(b)), i.e. the effect of thermal relaxation is relatively small at $100 \mathrm{kHz}$, and as a result hysteresis loops are broad and dominated by the metastability-driven switching events of MPs. On the contrary, the external field frequency $1 \mathrm{~Hz}$ is in the slow range dominated by thermal relaxation. The MP system at $1 \mathrm{~Hz}$ is close to the thermal equilibrium when the evolution of magnetisation states approaches the quasi-static limit through a sequence of equilibrium states, resulting in the observed narrow hysteresis loops. At both frequencies, the hysteresis loop shape depends on $D_{f}$, as shown in Figs. 4(a)-(b) and (c)-(d) respectively for $f_{h}=100$ $\mathrm{kHz}$ and $1 \mathrm{~Hz}$, although the dependence of the major hysteresis loop at $100 \mathrm{kHz}$ is only weak.

Fig. 5 shows systematic investigations of the dependence of hysteresis loop parameters on $D_{f}$, such as of the zero-magnetisation coercive field, $H_{c}\left(=H_{K} h_{c}\right)$, zerofield remanent magnetisation, $M_{r}$, and the hysteresis loop area, $A$, normalised by their respective equivalent non-interacting MP case values (0Int). Two external field orientations were assumed in calculations, namely along the $\hat{z}$ axis (circles in Fig. 5) and $\hat{x}$ axis (squares). Figs. 5(a1)-(a3) and (a4)-(a6) show, respectively, the $D_{f}$ dependence of the major and inner minor hysteresis loop characteristics for $f_{h}=100 \mathrm{kHz}$. All of the considered hysteresis loop characteristics $H_{c}, M_{r}$, and $A$ reduce with the increasing $D_{f}$, which is a clear indication of the effect of particle-particle interactions within clusters. In addition, while the changes in the behaviour with respect to the non-interacting case seem small in case of the major hysteresis loop at $f_{h}=100 \mathrm{kHz}$ (a1)-(a3), the changes for the minor hysteresis loop are considerable, including the effect of orientation of the external field $\vec{h}$. In particular, the particle-particle interactions inside MP clusters can result to as much as $50 \%$ reduction of the hysteresis loop area, which is consistent with recent experimental observations. ${ }^{14}$

Similar observations are seen for reduced frequency 1 $\mathrm{Hz}$ in Fig. 5(b1)-(b6). In this case, due to the more pronounced similarity of the major and minor loops, the behaviours seen in figures (b1)-(b3) and (b4)-(b6) are similar. There is a clear significant reduction in the magnitudes of the hysteresis loop parameters as the $D_{f}$ increases, which implies increasing relative differences with respect to the non-interacting case.

\subsection{Fourier analysis of time-dependent magnetisation}

Fig. 6(a) shows the plot of time-dependent magnetisation for the minor hysteresis loop given in Fig. 4(a). The corresponding discrete Fourier transform is shown in Fig. $6(\mathrm{~b})$. The dominant peak in the frequency spectrum is the first harmonic with magnitude $\tilde{M}_{1}$, located at the driving field frequency $f_{h}=100 \mathrm{kHz}$. The next largest peak is the $3 r d$ harmonic with frequency $3 f_{h}$ and magni- 

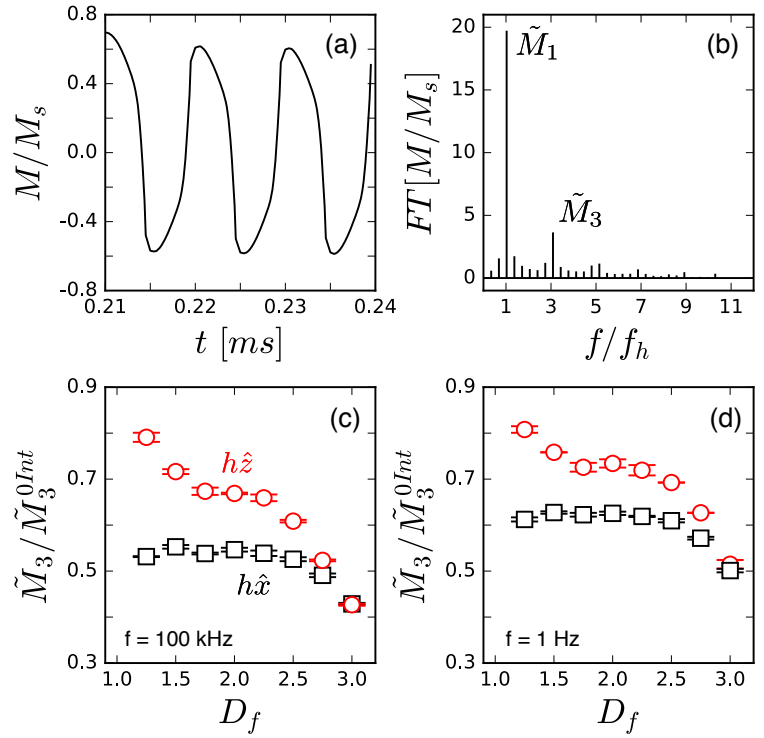

FIG. 6. (a) Time-dependence of magnetisation of the minor loop cycle shown in Fig. 4(a). (b) Discrete Fourier transform of the magnetisation time series shown in (a), with the frequency axis normalised by the frequency of sinusoidal driving field $f_{h}=100 \mathrm{kHz}$. (c)-(d) Fractal dimension dependence of the magnitude of the 3rd harmonic frequency, $\tilde{M}_{3}$, for $f_{h}$ $=100 \mathrm{kHz}$ (c) and $1 \mathrm{~Hz}$ (d). The magnitudes of the 3rd harmonic frequency corresponding to non-interacting particle case where $\tilde{M}_{3}^{\text {oint }}=4.52$ for $f_{h}=100 \mathrm{kHz}$, and $\tilde{M}_{3}^{\text {0int }}=5.37$ for $f_{h}=1 \mathrm{~Hz}$.

tude $\tilde{M}_{3}$. Since the signal of the 3 rd harmonic can be distinguished from the driving field, it is this harmonic that is conveniently detected in Magnetic Particle Imaging ${ }^{3,4}$.

Fig. 6(c) shows a decreasing trend of the dependence of $\tilde{M}_{3}$ on the fractal dimension $D_{f}$ for $f_{h}=100 \mathrm{kHz}$, assuming the normalisation by the 3rd harmonic of the non-interacting case $\tilde{M}_{3}^{\text {OInt }}=4.52$. The behaviour for low $D_{f}$ is dependent on the external field orientation $\vec{h}=h \hat{z}$ or $\vec{h}=h \hat{x}$, and becomes independent of the field orientation when $D_{f} \sim 3$, due to the spherically symmetric geometry of clusters. Furthermore, the reduced ratio suggests that the magnitude of $\tilde{M}_{3}$ is considerably smaller in comparison to the non-interacting case $\tilde{M}_{3}^{0 I n t}$. Qualitatively similar observations hold for external fields with low frequency $f_{h}=1 \mathrm{~Hz}$ shown in Fig. 6(d).

\section{Discussion}

The studies of magnetic relaxation and hysteresis loops presented in Sections 4 and 5 share several similar features: i) the monotonic dependence of the behaviour on the fractal dimension $D_{f}$, where as $D_{f}$ increases the thermal relaxation monotonically accelerates and the size of hysteresis loops reduces, ii) the effect of the external field orientation for ensembles with lower $D_{f}$, and iii) the systematic reduction of the quantitative magnetisation characteristics with respect to equivalent systems of non-interacting MPs, such as the hysteresis loop coercivity, remanence and area.

Future studies will be required to fully understand the precise physical mechanisms behind these findings. As a simple qualitative argument, it is convenient to express the energy barrier $\Delta E_{\alpha \beta}$ for a transition $\beta \rightarrow \alpha$ via the reversal of a particle moment $\hat{m}_{k}$ inside a cluster using the approximation: ${ }^{33}$

$$
\Delta E_{\alpha \beta} \sim\left(1-\frac{\left|\vec{h}_{k}^{\mathrm{dip}}\right|}{\Theta}\right)^{\Gamma}
$$

where $\vec{h}_{k}^{\text {dip }}$ is given by Eq. (2), and $\Gamma$ and $\Theta$ take values, respectively, between 1-2 and 0.2-1 depending on the relative orientation between the particle anisotropy axis and the applied field direction. ${ }^{33}$ Expressing the $\left|\vec{h}_{k}^{\text {dip }}\right|^{2}=\vec{h}_{k}^{\text {dip }} \cdot \vec{h}_{k}^{\text {dip }}$ using Eq. (2) gives:

$$
\begin{aligned}
\left|\vec{h}_{k}^{\mathrm{dip}}\right|^{2}= & I^{2} \sum_{i \neq k} \sum_{j \neq k} \frac{1}{r_{k i}^{3} r_{k j}^{3}} \times \\
& \left(\hat{m}_{i} \cdot \hat{m}_{j}-6\left(\hat{m}_{i} \cdot \hat{r}_{k i}\right)\left(\hat{m}_{j} \cdot \hat{r}_{k i}\right)\right. \\
& \left.+9\left(\hat{m}_{i} \cdot \hat{r}_{k i}\right)\left(\hat{m}_{j} \cdot \hat{r}_{k j}\right)\left(\hat{r}_{k i} \cdot \hat{r}_{k j}\right)\right)
\end{aligned}
$$

As the fractal dimension $D_{f}$ of clusters increases, the number of neighbours of a MP increases accordingly (Fig. 2(c)), including the extent of randomisation of MP positions when the vectors $\hat{r}_{k i}$ eventually begin to point in all directions as $D_{f}$ increases. There is a significant cancelation tendency in the second and third term in the summation in Eq. (13), and the magnitude $\left|\vec{h}_{k}^{\mathrm{dip}}\right|$ and consequently the energy barrier in Eq. (12) become fully determined by the first term in Eq. (13). It is then evident, that in a MP system with spherically random distribution of uniaxial anisotropy, such as considered in this study, there cannot be any intrinsic preferential direction to compete with any particular external field orientation and all field directions must be equivalent in the limit of high $D_{f}$, which consistent with Figs. 3-6. On the other hand, in clusters with low $D_{f}$, there are only a few local neighbours of a MP, the position vectors $\hat{r}_{k i}$ are locally relatively well ordered, and as a result the cancelation tendencies of the individual terms in Eq. (13) are less likely. This gives rise to preferential directions due to the particular arrangement of MPs within clusters, even in systems with spherically random anisotropy axes, which leads to the dependence of the magnetisation behaviour on the external field direction, as can again be seen in Figs. 3-6 in the limit of low $D_{f}$.

The cluster size dependence has also been considered by assuming ensembles of clusters with reduced number of MPs to $N=20$ and 50 and yielded similar results (not shown). This suggests robustness of the observed behaviour. However, finite size effects are to be expected after reducing the clusters size to $N<10 .^{28}$ 
From the perspective of theranostic technologies, there are several implications of these findings. First the decreased hysteresis loop area between the interacting and non-interacting MPs seen in Fig. 5 implies the reduction of the specific heat loss in MP hyperthermia, which is consistent with earlier experiments demonstrating the effects of MP aggregation. ${ }^{14-16}$ The extent of this reduction is strongly dependent on the fractal nature of aggregates, and the direction, amplitude, and frequency of the applied field. The reduction of the magnitude of $\tilde{M}_{3}$ with the increasing fractal dimension of aggregates, relative to the non-interacting case $\tilde{M}_{3}^{\text {0int }}$, which is shown in Fig. 6, has important implications for Magnetic Particle Imaging. The signal-to-noise ratio in Magnetic Particle Imaging is directly proportional to $\tilde{M}_{3},{ }^{34,35}$ implying that the effect of dipolar interactions in randomised MP aggregates with fractal structure is to reduce the quality of detection. The field orientation-dependent differences in the relaxation behaviour shown in Fig. 3 could be exploited for developing multiplexed sensing and detection methodologies based on magneto-relaxometry. ${ }^{32}$

Finally, the statistical distributions of the intrinsic properties of MPs, such as size distributions, not considered in the present study, are also expected to have an important effect on the overall behaviour and will be investigated in the future. The effects of the external field waveform on hysteresis loops, such as a triangular or square waveform, also needs to be explored, as there is a preliminary evidence of significant changes in the hysteresis behaviour. ${ }^{36,37}$ Additional questions of important significance include the need for gaining new understanding of the details of statistical distributions of fractal dimensions of MP aggregates within cells such as evidenced recently, ${ }^{16}$ or whether certain cell types lead to aggregates with preferred fractal dimensionality. Orientational alignment of clusters inside cells under different experimental conditions also remains to be systematically studied prior full optimisation of theranostic applications can be achieved.

\section{Conclusions}

In conclusion, this article presents extensive kinetic Monte-Carlo study of thermally activated magnetisation dynamics in clusters of statistically distributed magnetic nanoparticles with relevance to theranostic applications. The computed magnetisation relaxation decay and frequency-dependent hysteresis loops have been found to significantly depend on the fractal dimension of aggregates, leading to accelerated magnetisation relaxation and reduction in the hysteresis loops size with the increasing fractal dimension of aggregates. These findings have implications for theranostic applications in nanomedicine, such as magnetic hyperthermia, magnetic particle imaging, and sensing and detection based on magneto-relaxometry.

\section{Acknowledgements}

The author would like to acknowledge fruitful discussions with Sergiu Ruta, David Serantes, Chris Hogan, and Roy
Chantrell, and thank the organisers of the 9-th International Conference on Fine Particle Magnetism 2016 (NIST, Gaithersburg) for their kind invitation, which helped to drive the preparation of this article.

${ }^{1}$ V. I. Shubayev, T. R. Pisanic, and S. Jin, Advanced Drug Delivery Reviews 61, 467 (2009).

${ }^{2}$ E. A. Périgo, G. Hemery, O. Sandre, D. Ortega, E. Garaio, F. Plazaola, and F. J. Teran, Applied Physics Reviews 2, 041302 (2015).

${ }^{3}$ B. Gleich and J. Weizenecker, Nature 435, 1214 (2005).

${ }^{4}$ N. Panagiotopoulos, R. L. Duschka, M. Ahlborg, G. Bringout, C. Debbeler, M. Graeser, C. Kaethner, K. Lüdtke-Buzug, H. Medimagh, J. Stelzner, T. M. Buzug, J. Barkhausen, F. M. Vogt, and J. Haegele, International Journal of Nanomedicine 10, 3097 (2015).

${ }^{5}$ C. Sun, J. S. Lee, and M. Zhang, Advanced Drug Delivery Reviews 60, 1252 (2008).

${ }^{6}$ F. Wiekhorst, U. Steinhoff, D. Eberbeck, and L. Trahms, Pharmaceutical research 29, 1189 (2012).

${ }^{7}$ S. J. Osterfeld, H. Yu, R. S. Gaster, S. Caramuta, L. Xu, S.-J. Han, D. A. Hall, R. J. Wilson, S. Sun, R. L. White, R. W. Davis, N. Pourmand, and S. X. Wang, Proceedings of the National Academy of Sciences 105, 20637 (2008).

${ }^{8}$ L. Gutierrez, R. Costo, C. Gruttner, F. Westphal, N. Gehrke, D. Heinke, A. Fornara, Q. A. Pankhurst, C. Johansson, S. Veintemillas-Verdaguer, and M. P. Morales, Dalton Trans. 44, 2943 (2015).

${ }^{9}$ C. Martinez-Boubeta, K. Simeonidis, D. Serantes, I. CondeLeborán, I. Kazakis, G. Stefanou, L. Peña, R. Galceran, L. Balcells, C. Monty, et al., Advanced Functional Materials 22, 3737 (2012).

${ }^{10}$ R. Hao, R. Xing, Z. Xu, Y. Hou, S. Gao, and S. Sun, Adv Mater 22, 2729 (2010).

${ }^{11}$ R. Otero-Lorenzo, M. C. Weber, P. A. Thomas, J. Kreisel, and V. Salgueirino, Phys. Chem. Chem. Phys. 16, 22337 (2014).

${ }^{12}$ C. Blanco-Andujar, D. Ortega, P. Southern, Q. A. Pankhurst, and N. T. K. Thanh, Nanoscale 7, 1768 (2015).

${ }^{13}$ D. Cabrera, J. Camarero, D. Ortega, and F. J. Teran, Journal of Nanoparticle Research 17, 1 (2015).

${ }^{14}$ R. D. Corato, A. Espinosa, L. Lartigue, M. Tharaud, S. Chat, T. Pellegrino, C. Ménager, F. Gazeau, and C. Wilhelm, Biomaterials 35, 6400 (2014).

${ }^{15}$ M. L. Etheridge, K. R. Hurley, J. Zhang, S. Jeon, H. L. Ring, C. Hogan, C. L. Haynes, M. Garwood, and J. C. Bischof, Technology 2, 214 (2014).

${ }^{16}$ S. Jeon, K. R. Hurley, J. C. Bischof, C. L. Haynes, and C. J. Hogan, Nanoscale 8, 16053 (2016).

${ }^{17}$ D. Soukup, S. Moise, E. Céspedes, J. Dobson, and N. D. Telling, ACS Nano 9, 231 (2015).

${ }^{18}$ I. Andreu, E. Natividad, L. Solozábal, and O. Roubeau, ACS Nano 9, 1408 (2015).

${ }^{19}$ R. W. Chantrell, N. Walmsley, J. Gore, and M. Maylin, Phys. Rev. B 63, 024410 (2000).

${ }^{20}$ R. P. Tan, J. Carrey, and M. Respaud, Phys. Rev. B 90, 214421 (2014).

${ }^{21}$ S. Ruta, R. Chantrell, and O. Hovorka, Sci. Rep. 5, 9090 (2015).

${ }^{22}$ W. F. Brown, Phys. Rev. 130, 1677 (1963).

${ }^{23}$ J. Brown, W., Magnetics, IEEE Transactions on 15, 1196 (1979).

${ }^{24}$ N. G. Van Kampen, Stochastic processes in physics and chemistry, Vol. 1 (Elsevier, 1992).

${ }^{25}$ A. P. J. Jansen, An introduction to kinetic Monte Carlo simulations of surface reactions, Vol. 856 (Springer, 2012).

${ }^{26}$ To maintain the mathematical consistency with Master Equation Eq. (4) the Metropolis update rules for small energy barriers proposed in Ref. ${ }^{19}$ are not considered in this work, which makes the approach equivalent to Ref. ${ }^{20}$.

${ }^{27}$ While the moments of all other MPs in a cluster are adjusted smoothly in consistency with the local energy minimum $E_{\alpha}$ of the state $\alpha$. 
${ }^{28}$ O. Hovorka, J. Barker, G. Friedman, and R. W. Chantrell, Phys. Rev. B 89, 104410 (2014).

${ }^{29}$ G. Vallejo-Fernandez and K. O'Grady, Applied Physics Letters 103, 142417 (2013).

${ }^{30}$ G. Vallejo-Fernandez, O. Whear, A. G. Roca, S. Hussain, J. Timmis, V. Patel, and K. O'Grady, Journal of Physics D: Applied Physics 46, 312001 (2013).

${ }^{31}$ A. Filippov, M. Zurita, and D. Rosner, Journal of Colloid and Interface Science 229, 261 (2000).

${ }^{32}$ O. Laslett, S. Ruta, J. Barker, R. W. Chantrell, G. Friedman, and O. Hovorka, Applied Physics Letters 106, 012407 (2015).

${ }^{33}$ H. Pfeiffer, physica status solidi (a) 118, 295 (1990).

${ }^{34}$ R. M. Ferguson, K. R. Minard, and K. M. Krishnan, Journal of Magnetism and Magnetic Materials 321, 1548 (2009).

${ }^{35}$ R. M. Ferguson, K. R. Minard, A. P. Khandhar, and K. M. Krishnan, Medical Physics 38, 1619 (2011).

${ }^{36}$ S. M. Morgan and R. H. Victora, Applied Physics Letters 97, 093705 (2010).

${ }^{37}$ S. M. Morgan, H. Sohn, and R. H. Victora, Journal of Applied Physics 109, 07 (2011). 